\title{
SIMPLIFYING SOME THERMAL CALCULATIONS BY THE USE OF THE THERMAL OHM.
}

BY

CARL HERING, M.E.,

Cousulting Electrical Engineer.

If a manufacturer is using a machine which does its work in a slow, intricate, round-about, complicated manner, necessitating that considerable attention be given to it, he would, if he were progressive, be very glad to adopt a new one, if it turned out exactly the same product but in a simpler, more direct, and more reliable way, with economy of labor and attention, and he would willingly, scrap the old one, especially if the new one were presented to him. Yet when the machine is the man's own brain, and the work it does is to exert mental effort in performing calculations requiring thought and care, and involving at times great responsibilities, the average man will continue to work in the rut dug by his forefathers, rather than to adopt improvements that are offered to him gratis, which have for their object economy in brain work, or what may be called the conservation of mental effort.

As an illustration, a vast amount of such mental effort is being absolutely wasted daily by. millions of people in Great Britain by the use of their senseless, antiquated, irrational but beloved $\mathfrak{t} \mathrm{s}$. $\mathrm{d}$. We in this country could appreciate it, if we were to think for a moment of what a mental tax it would be to us, in our daily finances, to change back from our decimal system to the awkward, antiquated English one. Similarly, the people in the more progressive nations like Germany and France, who have adopted the decimal system in all their other measures, can use for other and really profitable purposes the vast amount of brain labor and mental effort which we in this country are wasting daily with our awkward and cumbersome system of weights and measures, which they look upon as being as absurd as we think the English money system is. It is no wonder, therefore, that such magnificent mental work issues from those 
more progressive countries in which man's brain is not hampered and fatigued by the wasteful mental brake which it has applied to it incessantly, as it is in this country and even to a still greater extent in England.

In an article in the February, I9II, issue of this journal, page I29, the writer made an analysis of systems of measures and called attention to numerous improvements that could be made with a view to economy in mental effort in performing calculations such as an engineer has to make daily. It was there shown that the physicist has the great advantage in using the so-called absolute or c.g.s. system, in which there is one, and only one, unit for each physical quantity, and in which nearly all coefficients and conversion factors drop out of the calculations because they are unity; and that it would be quite feasible for the engineer to benefit by the use of a similarly ideally simple system by merely adopting as his working units decimal multiples or fractions of the absolute units, as in fact the electrical engineer has done in his excellent brain-saving system of electrical units. It would even be of more importance to the engineer than the absolute system is to the physicist, because he has vastly more calculations to make, and they are often of greater importance, as mistakes in them in designing engineering structures may cost the lives of innocent people.

Although such means for economizing mental effort are offered to us gratis, the average man is like the manufacturer who prefers to continue to use antiquated machinery rather than to scrap it and accept the better onc that he can obtain without cost. He complains that it is too much trouble for him to move away the old machine and set up the new one, as his more progressive neighbor does. The natural result is that the one gets the trade while the other is fossilizing. It is not surprising, therefore, that, in accordance with the law of the survival of the fittest, a progressiva country like Germany, where the economy of mental effort enables that effort to be more profitably applied, is fast getting the world's trade from its neighbor who is burdened with a fossilized system of mental brakes in the shape of absurdly complicated, brain-robbing systems of measures, almost all of which we had the misfortune to inherit from England, and which we cling to as the unprogressive manufacturer clings to his antiquated, out-of-date machine. 
Although it may, unfortunately, take us several generations more before we succeed in giving up our cumbersome systems of measures, and adopting the decimal systems, yet there are some directions in which we can save mental effort without parting with any of our beloved antiquities, namely, by adopting some new and rational units or measures where none existed before.

The purpose of the present article is to show how, by the adoption of a new unit for a quantity for which no unit has existed, the mental labor in a certain class of calculations can be greatly simplified and reduced.

In general, when no definite unit exists, we naturally refrain from using that quantity by adopting some round-about methods, or, when we must use it, we have to define a unit of our owna home-made unit, as it were-which generally results in different writers giving their data in different units, thereby causing the waste of much mental effort by the one who desires to combine and use such data from different sources. In a recent case of this kind, an author of an otherwise excellent paper had made up such a home-made unit, quite different from all the others; in terms of which his valuable quantitative results were given, but had carelessly omitted to give the receipt according to which that complicated unit had been concocted, and it cost the present writer the best part of a day to work out its composition before the data became available, all of which mental effort could have been saved had the unit been described, or, better yet, had there existed a well-defined, recognized, international unit for measuring that quantity.

One of the groups of calculations in which no definite units exist for certain quantities is that concerned with the flow or transmission of heat by conduction, convection, and radiation. It therefore concerns such calculations as the thermal insulation of steam or refrigerating pipes, of electric and other furnaces, the transference of heat from the flames to the water in boilers, or from the flames or the arc of a furnace to the metal to be melted, the insulation of thermos bottles, the cutting of metals with flames; the repairing of broken metal machines by local fusion, etc.:

For measuring heat energy itself, statically, plenty of units exist; too many, unfortunately: the large calorie, the small 
calorie, the B.T.U., the mongrel pound-centigrade unit, the joule, and the erg, some of which have several names; in name there exists a seventh, the "heat unit," which may mean nearly any of the others, according to the fancy of the writer, and is therefore meaningless unless defined by the user.

When a flow of heat is involved, that is, a rate of transmission of heat, the element of time must be introduced, and here there is again the choice of the hour, minute, or second, according to the fancy of the writer. Six heat units combined with three time units make eighteen possible units of heat flow, from among which different writers seem to delight in choosing that one which they think other writers have slighted. However, each onc of this family of eighteen would be an intelligible unit for expressing a flow of heat if its particular make-up is clearly specified by its particular admirer.

Flows of heat through bodies are conducted, and they then meet with more or less resistance in that body, which resistance, for instance, is said to be about twenty or thirty thousand times as great through a piece of flannel as it is through an equal piece of copper. And as this thermal resistance varies so greatly and is of great importance, it becomes very desirable, and sometimes even necessary, to use it as a quantity capable of being measured, calculated, and specified; hence there should be a unit in terms of which this can be done. But no such unit existed, and the consequence is, as usual, that an author either evades using this quantity, or else makes up a unit of his own; this results in a great multiplicity of units ambiguity in their meaning, and, in general, much wasted mental effort on the part of the one who has to apply the data to practical cases. Some authors seem to take particular pleasure and pride in making up new composite units in such cases, and sometimes the only originality in an article is the invention of a different unit which no one else has cared to use.

Thermal resistances have in the past been generally avoided by using the reciprocals instead, namely, conductances (often incorrectly called conductivities, which are really specific conductances). But in by far the most cases in practice a flow of heat passes through several bodies in succession, that is, in series, as the electrical engineer terms it. Even in simple cases there are generally several successive contact resistances in series with 
that of the material itself, and these are sometimes extremely high; in fact, at times the most important of all, as in steam boilers, for instance, in which by far the largest part of the virtual thermal resistance is at the contact of the flames and the outside of the water tubes; this is so very great that the difference between the resistance of copper and iron tubes (about 4 to I) falls into insignificance.

When heat or electricity flows through several bodies in series it is far simpler to use resistances in the calculations, instead of conductances; and when they are in multiple the reverse is the case. The former being by far the more general case, it is much more rational to use thermal resistances than the older and more common conductances. This is the first of the improvements which is here recommended for the purpose of economizing mental effort in such calculations.

But in view of the fact that conductances have in the past been used almost exclusively, let us consider for a moment the question of the units of conductance and conductivity. Such units are composite and must be made up, and this particular case is a good illustration of how each writer is apt to make up one of his own without the slightest regard for uniformity. Moreover, it seems that no one, until recently, has ever suggested a unit of conductance itself, but only of conductivity. Notwithstanding the convenience which the former would be, let us consider here only the latter.

A unit of conductivity is defined by a certain flow of heat through a specified cross section for a specified length, hence involves areas and lengths. For the latter the physicists usually take the square centimetre and the centimetre, hence they specify the heat flow through a centimetre cube; yet the inch is also often used, and until we have abandoned this irrational unit it is convenient to give conductivities in inch cube units also. Besides these two, some writers also use the metre cube, others the square foot combined with one inch thickness, etc.; in one noted case of an otherwise excellent article descriptive of some valuable research work, the author of it even introduced the inexcusable mongrel combination of one square metre area with one inch thickness. But assuming that there are only four sets of these linear and superficial units, then with the 18 different possible units of heat flow mentioned above this makes 72 pos- 
sible units of thermal conductivity-enough for the most fastidious writer to choose from if he wants something different from what others use; something "original."

This absurd and deplorable state of affairs, and the waste of mental effort which it involves, led the writer some time ago to investigate the subject with a view to finding, if possible, that unit in each case which is at the same time the most rational and reduces the calculations to the least possible.

As stated before, the units used in the absolute system are those which bring nearly all coefficients to unity, hence involve no troublesome conversion factors. Hence, whenever we are in want of a new unit it is best to go to this absolute system, and, as the units in that system are often inconveniently small or large, to take some decimal multiple of them. This is what was very wisely done when our system of electrical units was devised, and those who enjoy the simplicity of electrical calculations should be thankful to our forefathers for their good judgment and wisdom.

A flow of heat is a particular case of the transmission of energy, the particular one in which the transmitted energy is in the form of heat, but as it may be transformed from or into other forms of energy, it is best, in the selection of a unit, to consider energy in general, and not only one form of it. The unit of energy in the absolute system is the erg, which is equal to the force of a dyne acting through a centimetre. The unit of time in that system is always a second; hence the absolute unit for measuring a transmission of energy of any form is an erg per second. This being inconveniently small for practical work, it has been decided, for electrical purposes at least, to make the practical unit ten million times as great, and this unit is known as the watt.

Hence the most rational unit for measuring a flow of heat is the watt. To say that the heat flow in a certain case is soand-so-many watts means that the amount of heat is that which would be set free if that many watts were converted into heat, as in an electrical heater, for instance. Hence, if this unit were used, all coefficients and reduction factors would drop out (or be some multiple of Io), at least for all calculations with electrical quantities, such as electric furnaces or heaters, etc.

As a watt is a joule per second, the coefficients and con- 
version factors would also drop out in all our heat calculations if the tables of thermal constants were reduced once for all to joules. Specific heats, which are generally given relatively to water, would then be given by their true, absolute values in joules. But such a revolutionary change as that, which would involve the dropping of all four of our " home-made " units of heat, for which we seem to have such an affection, and using only the two natural or absolute ones, the erg and joule, is foreign to the present paper and need not be further discussed here.

Having found that the most rational unit of heat flow is the watt, and having decided that thermal resistance was a more generally useful quantity than conductance, the next question is, What is the correspondingly rational unit of thermal resistance? It is evidently that one which again reduces the coefficient to unity. The law of heat flow is, that the amount of flow increases in proportion with the temperature drop (which causes the flow) and decreases in proportion with the resistance (which opposes the flow); hence just like with electricity. This might be termed the thermal 'Ohm's law; or, represented by letters, if $W$ is the heat flow, $T$ the temperature drop, and $R$ the thermal resistance, then $W=T / R$. It is in this fundamental law that the coefficient is to be made unity.

In the absolute system of units there is no temperature scale, as temperature has not yet been reduced to absolute measure. Hence, until it is, we are compelled to use some one of the artificial temperature scales, and among these the centigrade is certainly the more rational and the most commonly used in most countries and to a gradually-increasing extent in this country.

Hence, as $T$ is to be in centigrade degrees and $W$ in watts, it follows that the unit for the thermal resistance $R$ must be so chosen that the coefficient in this law will be unity. Hence $R$ must be made equal to that thermal resistance which will allow, one watt to flow when the temperature drop is one degree centigrade; this is the unit that the writer has termed the thermal ohm, a name originally suggested by Dr. A. E. Kennelly, for thermal resistance, though for a quantitatively different unit.

Hence, when the physical property of a given body through which heat is flowing is stated in thermal ohms, and the temperature drop in centigrade degrees, the quotient of the latter by the former will give the heat flow in watts directly without 
any further reductions. It has therefore been reduced to the simplest possible kind of a calculation.

For calculations involving electric heat, such as those concerning electric furnaces and heating devices, nothing simpler could be desired, but even in other cases it will often be found to be simpler to make the calculations in thermal ohms and watts, and merely convert the final result back to the older and more cumbersome units, in which it may be desired.

The physical property of materials, called resistivity (specific resistance), as given in tables, is then stated in thermal ohms for one centimetre cube or one inch cube, and with the aid of these the total resistance of any given block of material is determined just as for electrical resistance, namely, by the usual formula $R=r L / S$, in which $R$ is the total resistance in thermal ohms, $r$ the resistivity in thermal ohms, $L$ the length and $S$ the section, centimetres or inches being used consistently throughout.

The use of thermal resistances as such, in calculations, is often a great convenience and time-saving device. For instance, in determining the insulating walls of electric furnaces the temperature drop is known, and so is also the allowable loss in the form of a percentage of the total input. The former divided by the latter then gives the resistance which the walls must have in thermal ohms. With the resistivities of the various materials and the inside dimensions of the furnace, the thickness of each layer of the different materials making up the total wall can then be calculated so that the sum of all the resistances is equal to that required. This would be an extremely tedious calculation to make with conductances. The temperature at the junction of any two layers can then also be readily calculated with the same formula; $W$ and $R$ being then the known quantities, $T$ is equal to their product.

In the insulation of steam pipes the temperature drop from the inside to the outside is known, and the permissible loss in power (reduced to watts) is decided upon; their quotient then gives the total insulation resistance in thermal ohms, hence per foot or per inch of pipe. Knowing the resistivities of the various insulating materials, the thickness of each can then be readily calculated, hence their respective costs, which can then be properly compared. It may thus be found, for instance, that it is cheaper to use a thin layer of a better insulator than a 
thick layer of a poorer one, or the reverse; or perhaps that a combination of two layers is better. For thick layers over small pipes the outer layers count for much less than the inner. ${ }^{1}$ Several such trial calculations would show to what extent the value of the steam energy which is being saved is worth the cost of saving it. In such cases the reduction to and from watts is made once for all, the simple Ohm's law being then available for the more numerous trial or comparative calculations.

For a certain limited class of simple calculations involving steam or fuel energy alone, when the physical constants are available in the best form for direct application, it may be more convenient to use the older method based on conductivities and without reducing to watts; but when anything different from the ordinary is involved, it will probably be found to be simpler to perform the computations in terms of watts and thermal ohms, and reduce merely the end results to the units desired, just as it is often simpler, in the more involved calculations, to reduce values to the metric system, perform the calculations in that system, and convert the end result back to our old units. It is also possible, of course, to establish other units of thermal resistance, based on the particular thermal temperature, time and length units, which one may be accustomed to using, by a method which reduces the coefficient to unity, similar to the one here used to establish the thermal ohm.

Another advantage of such a unit as the thermal ohm is that, being based on the absolute system, it is a rational one to which all values determined in other units could be cxpected to be reduced by the authors, hence it could be used as a sort of standard international thermal unit in terms of which all determinations can be readily compared, and from which they can readily be reduced to any other specific units desired; a sort of clearing-house for the 72 possible units of resistance.

Attention is here called to the fact that in not a few books and papers the heat flow and thermal conductivity units based on the gramme calorie per second, centigrade, and centimetre cube are claimed to be the absolute units. This is an error, although a very common one; this can be shown by the fact that they are incommensurate with the true absolute units. There

${ }^{1}$ See an article by the present writer in the Electrical World, November 25, 19II, page 1303 . 
is no heat unit in the absolute system; heat is energy, and the energy unit in that system is the erg and not the gramme calorie. The units recommended by the writer (watt and thermal ohm) are the ones which are the decimal multiples of the true and correct absolute units, and they are incommensurate with the so-called absolute units just described.

A table of about 150 values of the thermal resistivities of different materials, taken from various sources and reduced to thermal ohms by the writer, is being published in the December issue of Metallurgical and Chemical Engineering. The values in centimetre cube units range from about 0.24 to about 3 for the metals; 25 to 180 for various bricks and solid stones; and from about 250 to 5000 for granular or fibrous materials mixed with air. It will therefore be seen that the unit is of a convenient size.

A table of conversion factors for reducing the various units of thermal resistances, conductances, resistivities and conductivities to one another is given in the writer's paper on the thermal $\mathrm{ohm}$, in Metallurgical and Chemical Engineering, January, I9I I, page I 3 .

Density of Niton ("Radium Emanation"). R. W. GRAY and W. Ramsay. (Roy. Soc. Proc. Ser., H.84,536.) --The balance used to weigh the niton ( $\mathrm{RaEm})$ was sensitive to 2 micro-milligrams and acted on the same buoyancy principle as that of Steele and Grant, but certain modifications were introduced. Five experiments were made to determine the atomic weight of niton; the mean result was 223. The amount of helium produced by the niton and its products on complete disintegration to $\mathrm{RaD}$ was determined by weighing, and the result agreed closely with the disintegration theory, although about one-fourth of the helium had entered the walls of the weighing-tube and had to be expelled by heat and subsequently weighed. The experiments afford further proof of the disintegration theory on which the weight of niton should be 222.4 ( $\mathrm{Ra} 226.4-\mathrm{He} 4$ ).

Temperature of the Sun. F. Kurlbaum. (Preuss. Akad. Wiss. Ber., $\mathrm{xxv}, 54 \mathrm{I}$.) - By a spectroscopic comparison of sunlight with a standard lamp, a series of determinations of the solar temperature have been made. The mean result is given as $5730^{\circ}$ abs. by the Holborn-Day scale or $6390^{\circ}$ abs. by the Holborn-Valentiner scale. 